\title{
Homogeneous Photoalignment of Liquid Crystals without Precoated Alignment Layers
}

\author{
Koichi Kotera ${ }^{1}$, Mizuho Kondo ${ }^{1}$, Kohei Goto ${ }^{2}$, Satoshi Minami ${ }^{2}$, Tomoyuki Sasaki ${ }^{3}, K_{0 h e i}$ Noda $^{3}$, \\ Hiroshi Ono ${ }^{3}$, and Nobuhiro Kawatsuki ${ }^{*}$ \\ ${ }^{1}$ Department of Applied Chemistry, Graduate School of Engineering, \\ University of Hyogo, Himeji, Hyogo 671-2280, Japan \\ ${ }^{2}$ Nissan Chemical Inc., 722-1 Tsuboi, Funahashi, Chiba 274-8507, Japan \\ ${ }^{3}$ Department of Electrical Engineering, Nagaoka University of Technology, \\ Nagaoka, Niigata940-2188, Japan \\ *kawatuki@eng.u-hyogo.ac.jp
}

\begin{abstract}
Homogeneously aligned liquid crystal (LC) cell was fabricated using nematic LCs (NLCs) doped with a polymerizable monomer comprising of photocrosslinkable mesogenic side groups, combined with the linearly polarized UV light exposure at elevated temperature. We have clarified that the photosensitive layer forms on the substrates via the thermal polymerization, followed by generating the NLC photoalignment based on the axis-selective photocrosslinking. The LC characteristics of the photosensitive side groups of the polymerizable material play an important role in the defect-free alignment for the NLCs.
\end{abstract}

Keywords: Photoalignment, Liquid crystal display, In-plane LC switching

\section{Introduction}

Because of its various advantages over the conventional rubbing method, photoalignment of nematic liquid crystals (NLCs) has been widely applied to the LC devise fabrication [1-5]. Generally, the precoated photoalignment layers on the substrates exposed to linearly polarized (LP) light are utilized to fabricate the LC cell [2-5]. Many types of photosensitive materials have been investigated for the photoalignment layer for LC devises [1-10]. In case of the photoalignment of NLCs after the cell fabricating, Yamaguchi et al. reported that the LP light exposure to a LC cell with precoated poly(vinyl cinnamate) (PVCi) photoalignment layers at the isotropic temperature range of the NLCs controlled the homogeneous alignment direction [11]. Further improvement for the LC device fabrication should include the NLC alignment method without the use of a precoated alignment layer, which can reduce the LC devise fabrication process.

Several studies show the homeotropic and/or homogeneous NLC alignment without the precoated alignment layers [12-18]. The photo-switching between homeotropic and homogenous NLC alignment was demonstrated by adding a surfactant with azobenzene-side groups to the NLCs [12]. A homogeneous photoalignment could be controlled by using rubbed, polyimide-coated substrates injected with a NLC doped with a small amount of photoalignable polymer [15]. We have achieved a homogeneous LC photoalignment without the precoated layer by using NLC doped with photoalignable low-molecular-weight LC materials, which form thin photoalignment layer on the substrates, exposed to LPUV light at the isotropic temperature range of the NLCs [16,17]. However, several defects were seen in the NLC alignment. Minoura et al also reported a similar homogeneous NLC photoalignment using NLC doped with photosensitive monomer [18]. In these cases, interaction between the NLCs and the photoalignment layer formed on the substrates plays an important role in the alignability of the NLC and the stability of the LC cell.

Herein, we report a new material for the homogeneous 
NLC photoalignment without precoated alignment layers, which contains photoalignable mesogenic groups combined with polymerizable end group. Due to the improved interaction between the photoalignment layer and NLCs, defect-free homogeneous NLC alignment is obtained. The mechanism of the formation of the photoalignment layer followed by the NLC's photoalignment is clarified. Finally, in-plane switching-mode (IPS) NLC cell is demonstrated.

\section{Experimental}

\subsection{Materials}

Thermally polymerizable monomers (M1 and M2) used in this study is shown in Fig. 1, where M1 comprises photocrosslinkable mesogenic side groups, while M2 is prepared for comparison that does not exhibit mesogenic characteristics. For the NLC, Merck ZLI-4792 (N 92 I) was used as received. The NLC and M1(M2) were completely miscible and the thermal property of the NLC/M1(M2) [99/1(wt/wt)] was similar to that of NLC.

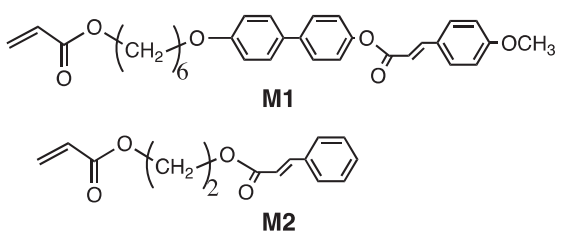

Fig. 1. Chemical structures of M1 and M2 used in this study.

\subsection{LC cell fabrication}

An LC cell was fabricated from a mixture of NLC and M1(M2) [99/1(wt/wt)] using glass substrates (Matsunami slide glass S1126), and those with a patterned electrode for IPS LC cell (EHC Co., ISSZ-10). A cell thickness of $6 \mu \mathrm{m}$ was attained using bead spacers, and $10 \mu \mathrm{m}$ for the IPS LC cell. After filling an empty cell with the mixture, the cell was heated at elevated temperature for 10-20 $\mathrm{min}$, followed by exposed to LP-313 nm light using a high-pressure $\mathrm{Hg}$ lamp equipped with Gran-Taylor prisms (intensity: $20 \mathrm{~mW} / \mathrm{cm}^{2}$ ). Then the cell was cooled to room temperature. The LC alignment performance and its direction were evaluated by doping with dichroic dye (DB14) with polarized optical microscopy (POM).

\section{Results and discussion}

\subsection{Homogeneously aligned LC cell}

Because M1 and M2 contain acrylate end groups, thermal polymerization occurs when the materials are exposed to elevated temperatures. The photoreactive side groups in M1 exhibits the same

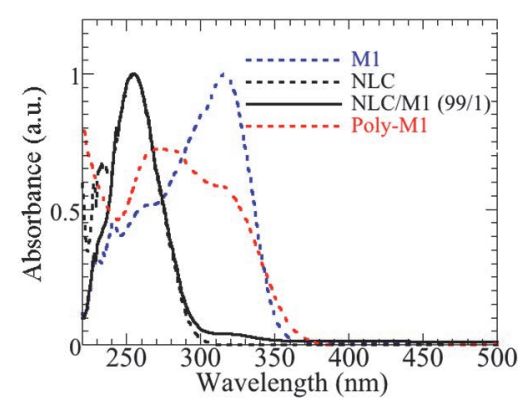

Fig. 2. UV-vis absorption spectra of NLC, M1, NLC/M1(99/1) mixture, and poly-M1 in chloroform solution.
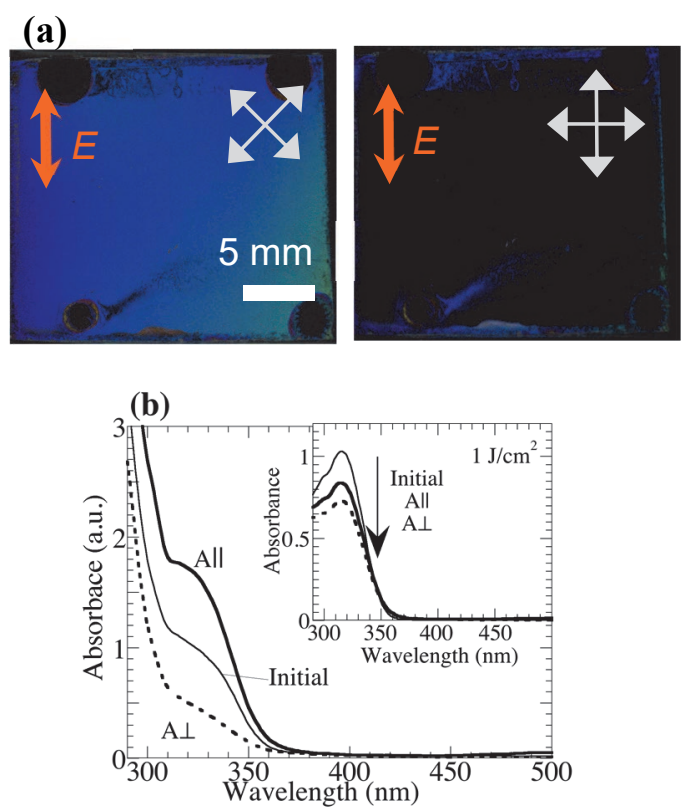

Fig. 3. (a) POM photographs of a LC cell fabricated from NLC/M1. White arrows indicate polarizer and analyzer directions, and red arrow indicates polarization of LP 313-nm light. (b) Polarized UVvis absorption spectra of a LC cell. Inset shows the polarized absorption spectra of M1 in PMMA film.

chemical structure to the photoalignable LC polymer reported previously, which can be available for the homogeneous NLC photoalignment layer [10]. Figure 2 plots the absorption spectra of NLC, NLC/M1, M1 and poly-M1 in chloroform solution, where the absorption band of M1 and poly-M1 is observed at $320 \mathrm{~nm}$.

Figure 3a shows POM photographs of a LC cell fabricated from NLC/M1 (99/1) preheated at $100{ }^{\circ} \mathrm{C}$ for $10 \mathrm{~min}$ followed by exposing to LP-313 nm light for $0.1 \mathrm{~J} / \mathrm{cm}^{2}$ at $100{ }^{\circ} \mathrm{C}$. It shows a clear homogeneous NLC alignment without defects in the exposed area. The NLC alignment direction is perpendicular to the polarization (E) of LPUV light, 
where the axis-selective photocrosslinking of M1 in the parallel direction to $\mathbf{E}$ induces the uniaxial NLC alignment. In the polarized absorption spectrum, the absorption band for the mesogenic side groups of M1 shows a significant dichroic ratio $\left[\mathrm{DR}\left(=A_{\|} / A_{\perp}\right)\right.$ at $320 \mathrm{~nm}=3.5$ ] (Fig. 3b), which is much larger than that obtained from M1 doped in PMMA for any exposure doses $\left(\mathrm{DR}_{\max }=1.2\right.$, inset of Fig $\left.3 \mathrm{~b}\right)$, suggesting the cooperative reorientation of M1 with the aligned NLC [10]. The estimated azimuthal anchoring energy using a Néel-wall method [19] was approximately $1.1 \times 10^{-4} \mathrm{~J} / \mathrm{m}^{2}$, which was larger than those using a photoreactive material without the polymerizable end groups and PVCi photoalignment layers $[16,17,19,20]$.

In contrast, many defects were seen for a NLC/M2 (99/1) cell even though homogeneous NLC alignment was obtained (Fig. 4). The calculated azimuthal anchoring energy was $1.5 \times 10^{-6}$ $\mathrm{J} / \mathrm{m}^{2}$. In this case, NLC alignment is conducted by the axis-selective photocrosslinking of the cinnamate moieties, which does not exhibit LC characteristics. These results indicate that the LC characteristics of the formed photoalignment layer, which is cooperatively aligned with NLC, play an important role in the uniform NLC alignment.
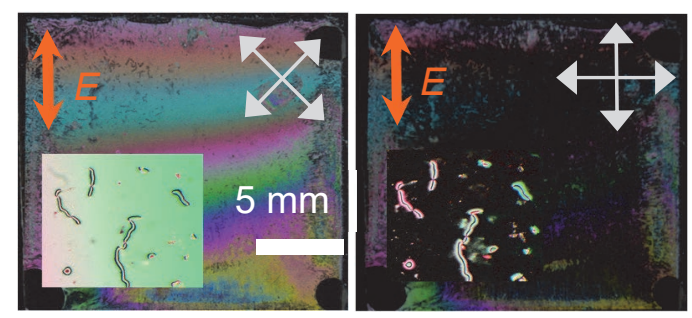

Fig. 4. Photographs of a LC cell fabricated from NLC/M2 under crossed polarizer. White arrows indicate polarizer and analyzer directions, and red arrow indicates polarization of LP-313 nm light. Inset shows Néel walls under POM observation.

\subsection{Photoalignment mechanism}

To elucidate the photoalignment layer formation, the pre-heated NLC/M1 (99/1) cell without the LP$313 \mathrm{~nm}$ light exposure was immersed into diethyl ether to remove the NLC and unpolymerized M1. Figure 5a plots the absorbance at $320 \mathrm{~nm}$ (absorption band of poly-M1 on the substrate) as functions of pre-hearting time at various heating temperatures. The absorbance increases when the heating time and temperature become longer and higher, respectively. By considering this result, the conversion of M1 for the photoalignment layer in the NLC/M1 cell (Fig. 3a) is estimated to be $5 \mathrm{~mol} \%$, suggesting that the non-polymerized M1 molecules remain in the cell. Additionally, post-annealing of the aligned NLC cell at the LC temperature range was effective to eliminate the monomeric M1. Figure 5b shows polarized absorption spectra of immersed substrates after the cell fabrication (same condition of Fig. 3a) and that with the post-annealing process at $90{ }^{\circ} \mathrm{C}$ for 1 day, showing the increased photoalignment layer thickness and slight orientation parallel to the LC alignment direction of the photoalignment layer.

These results indicate that the thermal polymerization of M1 molecules occurs at the preheating process, forming a thin layer on the substrates, and the subsequent axis-selective photoreaction of the thin film layer generates the NLC alignment. Controlling the pre-heating and post-annealing conditions decreases the M1 in the NLC.
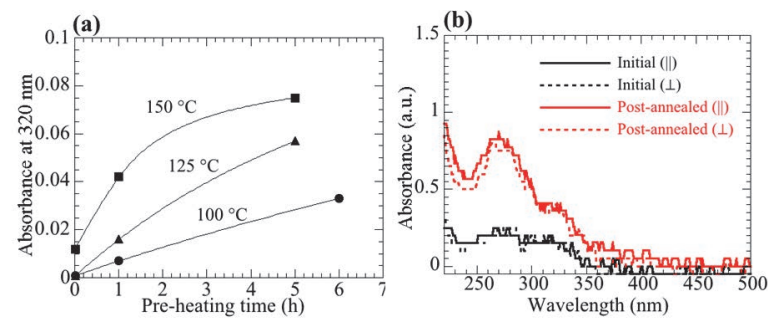

Fig. 5. (a) Change in the absorbance at $320 \mathrm{~nm}$ of the immersed substrates as functions of pre-heating time at various heating temperatures. (b) Polarized absorption spectra of an immersed substrate from a photoaligned NLC/M1 cell and that from a postannealed one at $90^{\circ} \mathrm{C}$ for 1 day.

\subsection{In-plane switching}

The electro-switching behavior of an IPS LC cell was evaluated using NLC/M1 (99/1) cell. Figure 6 shows POM photographs of a switching of the IPS LC cell, where NLC molecules adopted their initial alignment state when the applied voltage was turned on and off over 100 times, and $t_{\mathrm{on}(90 \%)}$ and $t_{\mathrm{off}(90 \%)}$ were 15 and $68 \mathrm{~ms}$ at $10 \mathrm{~V}(1 \mathrm{kHz})$, respectively. Additionally, the $t_{\mathrm{off}(90 \%)}$ became faster $(50 \mathrm{~ms})$ for a cell with post-annealing process. This is due to the increased azimuthal NLC anchoring caused by the molecularly oriented photoalignment layer as described in above section.

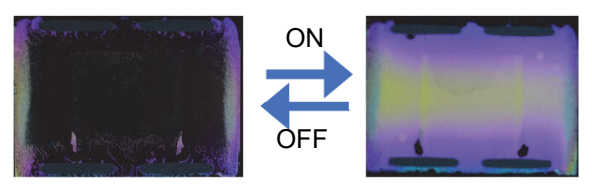

Fig. 6. Photographs of electro switching of a LC cell fabricated from NLC/M1 under crossed polarizer. 


\section{Conclusion}

The homogeneous photoalignment of NLCs without the precoated alignment layers was investigated using thermally polymerizable lowmolecular material with photocrosslinkable mesogenic groups doped in the NLCs. Thermal polymerization of the doped materials formed thin film on the substrates, followed by generating the axis-selective photocrosslinking of the mesogenic side groups exposed to the LP-313 nm light at the isotropic temperature range of the NLC. Thereafter, excellent NLC alignment is achieved due to the cooperative orientation of mesogenic side groups in the doped materials. For the defect-free NLC alignment, the doped polymerizable low-molecular material should comprises the mesogenic photoalignable side groups. This method can be applicable to reduce the fabrication process for the IPS-LC displays and functional LC devices.

\section{Acknowledgements}

This work was partially supported by a grant-inaid of Scientific Research B (No. 18H02039) and S (No. 16H06355) from the Japan Society for the Promotion of Science.

\section{References}

1. V. G. Chigrinov, V. M. Kozenkov, and H.-S. Kwok, "Photoalignment of Liquid Crystalline Materials" (Wiley, Chichester, U.K., 2008).

2. M. Schadt, H. Seiberle, and A. Schuster, Nature, 381 (1996) 212.

3. M. Schadt, K. Schmitt, V. Kozinkov, and V. Chigrinov, Jpn. J. Appl. Phys., 31 (1992) 2155.

4. M. Ibn-Elhaj, S. Chappellet, and F. Lincker, Solid State Phenom., 181-182 (2012) 3.

5. K. Goto, H. Yamanouchi, R. Ashizawa, A. Mandai, T. Nagi, S. Minami, K. Tsutsui, M. Ishikawa, M. Kondo, and N. Kawatsuki, SID
Symp. Dig. Tech. Pap., 44 (2013) 537.

6. O. Yaroshchuk and Y. Reznikov, J. Mater. Chem., 22 (2012) 286.

7. M. O'Neill and S. M. Kelly, J. Phys. D, 33 (2000) R67.

8. N. Kawatsuki, Chem. Lett., 40 (2011) 548.

9. U. V. Mahinly, A. I. Stankevich, A. A. Muravsky, and A. A. Murauski, J. Phys. D, 42 (2009) 075303.

10. N. Kawatsuki, K. Hamano, H. Ono, T. Sasaki, and K. Goto, Jpn. J. Appl. Phys., 46 (2007) 339.

11. R. Yamaguchi, A. Sato, and S. Sato, Jpn. J. Appl. Phys., 37 (1998) L336.

12. D.-Y. Kim, S. Kim, S. Lee, Y. Choi, W. Yoon, S. Kuo, C. Hsu, M. Huang, S. H. Lee, and K.-U. Jeong, J. Phys. Chem. C, 118 (2014) 6300.

13. O. Haba, D. Hiratsuka, T. Shirakawa, N. Funakoshi, H. Awano, T. Koda, T. Takahashi, K. Yonetake, M. Kwak, Y. Momoi, N. Kim, S. Hong, D. Kang, and Y. Choi, Opt. Mater. Express, 4 (2014) 934.

14. N. Kim, D.-Y. Kim, M. Park, Y. Choi, S. Kim, S.-H. Lee, and K.-U. Jeong, J. Phys. Chem. C, 119 (2015) 766.

15. T. Sasaki, T. Shoho, K. Goto, M. Sakamoto, K. Noda, N. Kawatsuki, and H. Ono, Appl. Phys. A, 122 (2016) 586.

16. N. Kawatsuki, Y. Hashimoto, M. Kondo, T. Sasaki, and H. Ono, Proc. IDW'15, (2015) 82.

17. Y. Hashimoto, M. Kondo, T. Sasaki, H. Ono, and N. Kawatsuki, Jpn. J. Appl. Phys., 56 (2017) 07170.

18. M. Mizusaki, H. Tsuchiya, and K. Minoura, Proc. IDW'17, (2017) 171.

19. K. Okubo, M. Kimura, and T. Akahane, Jpn. J. Appl. Phys., 42 (2003) 6428.

20. X. T. Li, D. H. Pei, S. Kobayashi, and Y. Iimura, Jpn. J. Appl. Phys., 36 (1997) L432. 